\title{
SCN4A variants and Brugada syndrome: phenotypic and genotypic overlap between cardiac and skeletal muscle sodium channelopathies
}

\author{
Véronique Bissay ${ }^{\star}, 1,7$, Sophie CH Van Malderen ${ }^{2,3,7}$, Kathelijn Keymolen ${ }^{4}$, Willy Lissens ${ }^{4}$, Uschi Peeters ${ }^{4}$, \\ Dorien Daneels $^{4}$, Anna C Jansen ${ }^{5}$, Gudrun Pappaert ${ }^{2}$, Pedro Brugada ${ }^{2}$, Jacques De Keyser ${ }^{1,6}$ and \\ Sonia Van Dooren ${ }^{4}$
}

SCN5A mutations involving the $\alpha$-subunit of the cardiac voltage-gated muscle sodium channel (NaV1.5) result in different cardiac channelopathies with an autosomal-dominant inheritance such as Brugada syndrome. On the other hand, mutations in SCN4A encoding the $\alpha$-subunit of the skeletal voltage-gated sodium channel (NaV1.4) cause non-dystrophic myotonia and/or periodic paralysis. In this study, we investigated whether cardiac arrhythmias or channelopathies such as Brugada syndrome can be part of the clinical phenotype associated with $S C N 4 A$ variants and whether patients with Brugada syndrome present with nondystrophic myotonia or periodic paralysis and related gene mutations. We therefore screened seven families with different SCN4A variants and non-dystrophic myotonia phenotypes for Brugada syndrome and performed a neurological, neurophysiological and genetic work-up in 107 Brugada families. In the families with an SCN4A-associated non-dystrophic myotonia, three patients had a clinical diagnosis of Brugada syndrome, whereas we found a remarkably high prevalence of myotonic features involving different genes in the families with Brugada syndrome. One Brugada family carried an SCN4A variant that is predicted to probably affect function, one family suffered from a not genetically confirmed non-dystrophic myotonia, one family was diagnosed with myotonic dystrophy (DMPK gene) and one family had a Thomsen disease myotonia congenita (CLCN1 variant that affects function). Our findings and data suggest a possible involvement of SCN4A variants in the pathophysiological mechanism underlying the development of a spontaneous or drug-induced type 1 electrocardiographic pattern and the occurrence of malignant arrhythmias in some patients with Brugada syndrome.

European Journal of Human Genetics (2016) 24, 400-407; doi:10.1038/ejhg.2015.125; published online 3 June 2015

\section{INTRODUCTION}

Sodium channel disorders are rare autosomal-dominant inherited diseases. They are caused by mutations in one of the subunit isoforms of voltage-gated sodium channels present in specific tissues such as the skeletal muscle, the cardiac muscle and the nervous system. ${ }^{1}$

SCN5A mutations involving the $\alpha$-subunit of the cardiac voltagegated muscle sodium channel $(\mathrm{NaV} 1.5)$ result in different cardiac channelopathies with an autosomal-dominant inheritance such as Brugada syndrome (BS). This syndrome is associated with syncope, life-threatening ventricular arrhythmias and sudden cardiac death. In the majority of patients with BS, no gene mutation is identified and only $30 \%$ of cases has an SCN5A mutation. ${ }^{2}$ The exact prevalence of this orphan disease is unknown, although it has been estimated to affect 1 in 2000 people worldwide. ${ }^{3}$

SCN4A encodes the $\alpha$-subunit of the voltage-gated sodium channel $(\mathrm{NaV1.4})$ in skeletal muscles. Mutations in this gene are responsible for muscular sodium channelopathies, encompassing (non-dystrophic) sodium channel myotonia (SCM), hyperkalemic periodic paralysis
(HyperPP), paramyotonia congenita (PMC) and a small percentage of hypokalemic periodic paralysis (HypoPP), as well as congenital myasthenia syndrome. The prevalence of muscular sodium channelopathies in the general population has been estimated to be $<1 / 100000{ }^{4}$

Except for nonspecific cardiac arrhythmias described in two SCN4A-associated case reports, ${ }^{5,6}$ no overlapping phenotypes between muscular and cardiac sodium channelopathies have been reported. Based on a personal observation of a patient with a genetically confirmed SCM and BS (patient A1 in Table 1), we investigated the possible role of SCN4A mutations in the pathophysiology of BS. The possible association between these two rare diseases is supported by several publications, demonstrating the expression of skeletal muscle voltage-gated sodium channels in the cardiac muscle. ${ }^{5,7,8}$ To determine whether cardiac arrhythmias or channelopathies such as BS can be part of an SCN4A-associated phenotype, we performed a cardiac work-up in the family of this patient as well as in six other families with an SCN4A variant. To increase the power of the tested hypothesis, a second cross-sectional

${ }^{1}$ Department of Neurology, Center for Neurosciences, UZ Brussel, Vrije Universiteit Brussel (VUB), Brussels, Belgium; ${ }^{2}$ Department of Cardiology, Heart Rhythm Management Center, UZ Brussel, Vrije Universiteit Brussel (VUB), Brussels, Belgium; ${ }^{3}$ Department of Electrophysiology, Thorax Center, Erasmus MC, Rotterdam, The Netherlands; ${ }^{4}$ Center for Medical Genetics, Reproduction and Genetics; Genetics and Regenerative Medicine, UZ Brussel, Vrije Universiteit Brussel (VUB), Brussels, Belgium; ${ }^{5}$ Pediatric Neurology Unit, Department of Pediatrics, UZ Brussel, Vrije Universiteit Brussel (VUB), Brussels, Belgium; ${ }^{6}$ Department of Neurology, University Medical Center Groningen, Groningen, The Netherlands

*Correspondence: Dr V Bissay, Department of Neurology, Center for Neurosciences, UZ Brussel, Vrije Universiteit Brussel (VUB), Laarbeeklaan 101, Brussels 1090, Belgium. Tel: +32 2 4763408; Fax: +32 2 4776800; E-mail: veronique.bissay@uzbrussel.be

7 These authors contributed equally to this work.

Received 22 August 2014; revised 3 April 2015; accepted 6 May 2015; published online 3 June 2015 


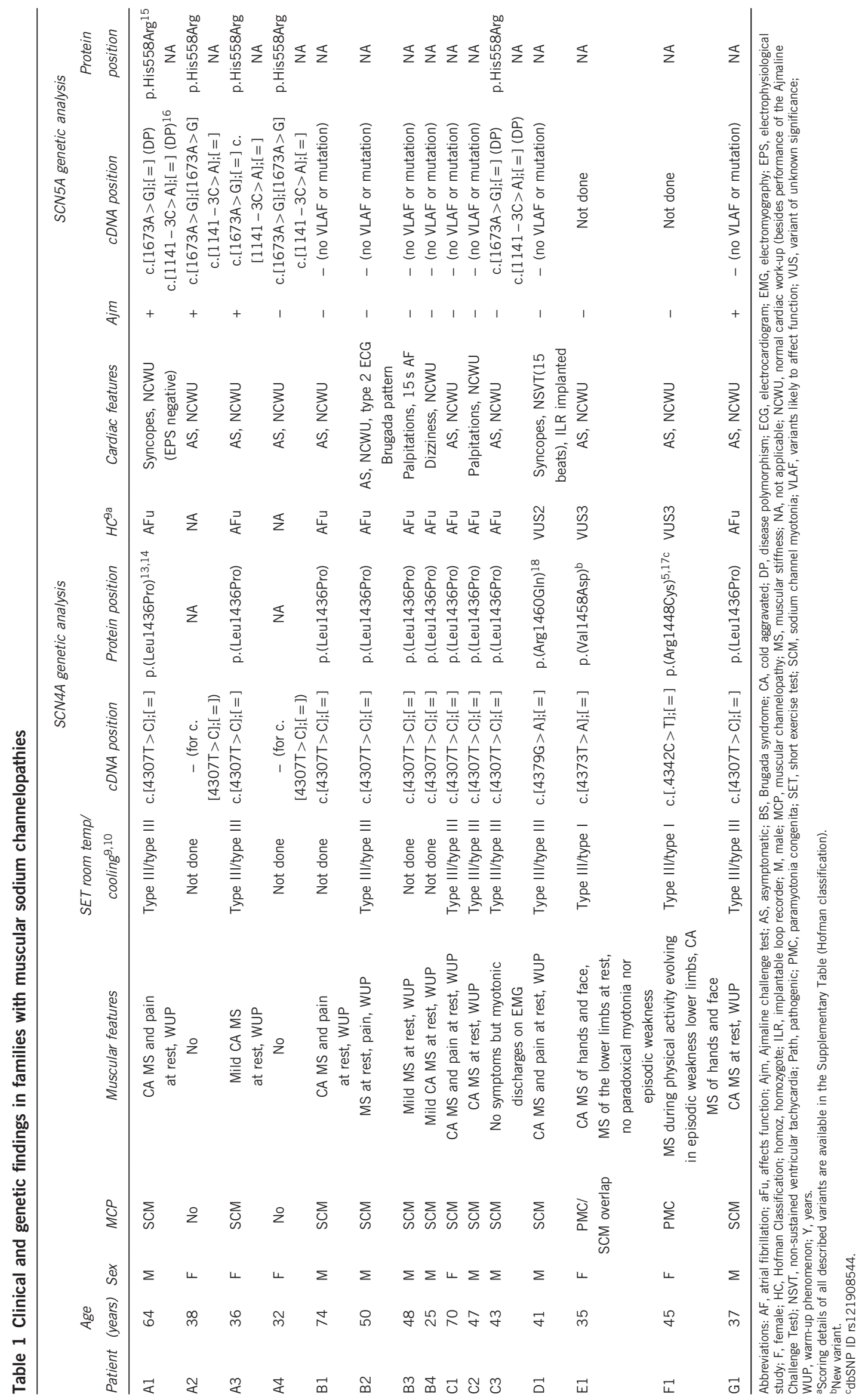


study was conducted to assess the prevalence of muscular sodium channelopathies in BS.

\section{MATERIALS AND METHODS}

Both studies were performed in accordance with the Declaration of Helsinki and approved by the Ethical Committee of the UZ Brussel. Written informed consent was obtained from all patients. All data have been submitted to the Leiden Open Variation database v.3.0 (http://chromium.liacs.nl/LOVD2/cancer/home.php). All identified variants are classified according to Wallis et al. and scored according to Hofman et al..$^{9,10}$ With these scoring list (scoring lists 1 for missense mutations and 2 for non-sense and frame-shift variants), the variants were classified into five different classes: no functional effect, variant of unknown clinical significance 1, 2 and 3 (VUS1, probably no functional effect; VUS2, unknown; VUS3, probably affects function) or affects function (for scoring details see Table 3 in Supplementary Material).

\section{First case/index family}

The hypothesis for this study was based on observations compiled from a 64-year-old male (patient A1 in Table 1). Electromyography (EMG) findings showed diffuse myotonia without myopathic features, and a short exercise test $^{11,12}$ suggested an SCM. Subsequent SCN4A analysis confirmed the presence of the c.4307T $>C$ variant p.(Leu1436Pro), a variant previously described by Matthews et al. ${ }^{13}$ Detailed description of the neurological symptoms of this patient and his family has been published elsewhere. ${ }^{14}$ One year earlier, the index was diagnosed with BS (Figure 1) after repeated syncopes and he underwent implantation of a cardioverter defibrillator (ICD). Genetic analysis showed no SCN5A variants that are likely to affect function. However, two polymorphisms (c.1673A > G (p.His558Arg) and c.1141-3C >A), described as possible disease-modifying variants, were found. These $S C N 5 A$ polymorphisms are also present in $20 \%$ of the general population. ${ }^{15,16}$

Consecutively, his three daughters (patient A2, A3 and A4) were screened for BS and both polymorphisms, regardless of the presence of the muscular channelopathy.

\section{First study: screening for BS in patients with $S C N 4 A$ variants}

Eleven neurologically affected members in six other unrelated families carrying an SCN4A variant (families B to G) underwent a cardiac work-up (Figure 2). Echocardiography demonstrated neither underlying cardiomyopathy nor structural heart disease in any patient. None of the included patients took any antiarrhythmic drugs at inclusion. The SCN4A phenotypes and genotypes of the different families are described in Table $1.5,9,13-18$ The diagnosis of BS was confirmed if patients had either a spontaneous or a drug-induced ST segment elevation with a type 1 morphology of $>2 \mathrm{~mm}$ in $>1$ lead among the right precordial leads (V1-V3) ${ }^{19}$ If no spontaneous type I morphology was seen on the electrocardiogram (ECG), a standardized Ajmaline Challenge Test was performed conforming to current guidelines to unmask any concealed forms of $\mathrm{BS}^{20}$ In case of established BS, SCN5A genetic analysis was performed, according to the recommendations from the Heart Rhythm Society/European Heart Rhythm Association. ${ }^{21}$ SCN5A variant detection in genomic DNA was carried out via high-resolution melting-curve analysis (HRMCA), followed by direct bidirectional Sanger sequencing analysis of aberrant HRMCA melting patterns and of exons and flanking intron regions for which HRMCA was not available. The interpretation was realized through SeqPilot v.4.0.1. using reference transcript NM_198056.2 and NG_008934.1. Other genes associated with BS, accounting for only $5 \%$ of all BS, were not screened in the myotonia probands.

\section{Second study: screening for muscular sodium channelopathy in patients with BS}

One hundred and sixty-nine adult BS patients of 107 Brugada families were recruited between October 2010 and March 2012 from the outpatient clinic of the UZ-Brussel. BS was previously diagnosed in the same manner as in the first study. In addition, a type C BS gene panel analysis of 16 extra BS-associated genes was performed in $S C N 5 A$-negative probands who consented for further genome-wide genetic testing. BS gene panel variants were detected via Roche
SeqCap v.3 target enrichment (Roche, Vilvoorde, Belgium) and 100 bp pairedend sequencing on an Illumina HiSeq1500 machine (Illumina, Eindhoven, The Netherlands). The in-house developed next-generation sequencing data analysis pipeline uses bwa v.0.7.10-r789, ${ }^{22}$ picard-tools v.1.97, ${ }^{23}$ samtools v.0.1.19, ${ }^{24}$ GATK v.2.75 and Alamut-HT v.1.1.11 (Interactive Biosoftware, Rouen, France). Detected variants that may affect function were confirmed by Sanger sequencing and SeqPilot v.4.0.1. (JSI Medical Systems, Ettenheim, Germany) data analysis.

Forty-two percent (71/169) of the patients received an ICD based on international recommendations at the time of implant. ${ }^{26}$ All patients underwent a clinical and electrophysiological assessment to detect a muscular sodium channelopathy (SCM, PMC, HyperPP and HypoPP) (Figure 2). They were interviewed by one of the investigators to assess the presence of episodic weakness and myotonia, the latter by asking about stiffness and pain, as well as alleviating and precipitating factors. All patients were tested for clinical myotonia, including evaluation of hand grip and lid lag, as well as eye closure and percussion myotonia over the thenar eminence. To detect the presence of myotonic discharges, seen in SCM and PMC, as well as sometimes in HyperPP, a standardized EMG was performed by an experienced neurophysiologist in all patients. This electrophysiological test was carried out at room temperature in a proximal and distal muscle of the upper and lower limb and after cooling of the hand muscle to $22^{\circ} \mathrm{C} .{ }^{18}$ The prolonged exercise test ${ }^{11}$ was only proposed in patients with a history of episodic muscle weakness, with the aim to confirm the phenotype of HyperPP or HypoPP.

Only in the presence of clinical and/or electrophysiological features suggesting a muscular sodium channelopathy, genetic testing of the $S C N 4 A$ gene was performed (Figure 2). Analysis for detection of SCN4A mutations was carried out by PCR amplification and Sanger sequencing of all 24 exons and parts (30 bp) of the flanking introns of the SCN4A gene (NM_000334.4 and NG_011699.1). In the absence of SCN4A variants that may affect function in patients with myotonic features, the CLCN1 (chloride channel, voltage sensitive 1, NM_000083.2 and NG_009815.1), which is the other gene involved in nondystrophic myotonia (NDM) was explored (Figure 2). ${ }^{27}$ CLCN1 was sequenced by PCR amplification and Sanger sequencing of all 23 exons and parts of the flanking introns. As NDM are considered to be highly penetrant genetic disorders, SCN4A or CLCN1 mutations are neither expected nor tested in the absence of clinical and/or electrophysiological features. ${ }^{27}$

One family was indicative for DMPK testing (Figure 2). Detection of the CTG repeat expansion in the $3^{\prime}$-UTR of the DMPK gene was performed with PCR techniques described by Brook et al. ${ }^{28}$ and with triplet repeat primed PCR techniques described by Warner $e$ e $a l .^{29}$ adapted for use on the ABI3130 genetic analyzer.

\section{RESULTS}

Screening for BS in patients with muscular sodium channelopathies In the index family (family $\mathrm{A}$ in Table 1), the three cardiac asymptomatic daughters (A2-A4) all carried the SCN5A polymorphisms c.1673A $>$ G (p.H558R) and c.1141-3C >A. Daughters A2 and A3 had a positive Ajmaline Challenge Test, revealing BS (Table 1 and Figure 1). During a subsequent electrophysiological study (EPS), no sustained ventricular arrhythmia could be induced in both patients. Therefore, ICD implantation was not indicated and follow-up once a year was proposed. One of both cardiac-affected daughters (A3) carried the same $S C N 4 A$ variant and the matching neurological phenotype as the father. The youngest daughter (A4) had normal cardiac tests and did not carry this SCN4A variant. One out of 11 patients of 6 additional families, all carrying an SCN4A variant, had a positive Ajmaline Challenge Test (Figure 1). No cardiac symptoms or suspicious family history was reported for this patient (G1). The subsequent EPS and SCN5A analyses of this patient were normal and no ICD implantation was proposed at that time.

One cardiac asymptomatic patient (B2) had a baseline Brugada type 2 pattern, which remained unchanged after the challenge test with ajmaline. This ECG pattern is therefore considered to be a normal 
variant rather than a specific predictor of life-threatening arrhythmia. $^{30}$

Extensive cardiac work-up in four patients (B3, B4, C2 and D1) with palpitations, syncope or dizziness only demonstrated the occurrence of a non-sustained ventricular tachycardia in one patient with syncope (D1; Table 1). Ajmaline testing and SCN5A analysis demonstrated no underlying cardiac channelopathy. All other patients had normal cardiac findings.

Screening for muscular sodium channelopathies in patients with BS Periodic paralysis was not diagnosed in any Brugada patient. Ten Brugada patients from four families had electrophysiological myotonic features (Table 2). In one family, a known SCN5A variant was identified and a second family carries a novel SCN5A variant. Both variants were predicted to affect function. ${ }^{2,31,32}$ In the other two families, no BS-associated variants were identified in SCN5A (H1) and the BS gene panel of 16 additional genes (I1). Clinical, neurophysiological features and genetic diagnoses are described in detail in Table 2. There was an important intra- and interfamilial variation in severity

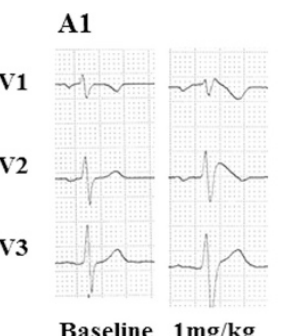

A3

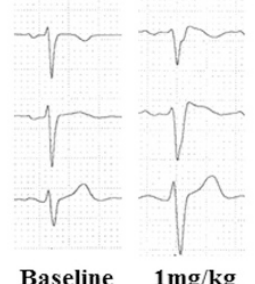

G1

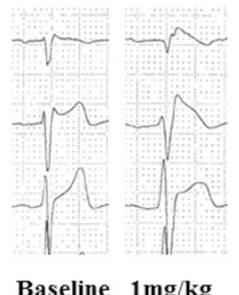

Figure 1 Right precordial ECG tracings (V1-V3) in three patients with an $S C N 4 A$-associated non-dystrophic myotonia and Brugada syndrome. The baseline ECG pattern in patient $A 1$ and $A 3$ is normal. Patient G1 demonstrates a type 2 baseline ECG (not diagnostic). After a maximal administered dose of ajmaline $(1 \mathrm{mg} / \mathrm{kg})$, baseline ECG patterns are converted into a diagnostic type 1 ECG pattern, which consist of a covedtype ST segment elevation. and distribution of myotonic features (Table 2 and Figure 3). Four out of ten patients (H1, I1, I3 and K1) had typical symptoms of myotonia and one (K1) had objective myotonic signs during physical examination. None of them had consulted a physician for these complaints in the past, suggesting a relatively mild impact on their quality of life.

In the proband of the first SCN5A-negative Brugada family (H1), a c.2341G >A p.(Val781Ile) in exon 4 of SCN4A was detected. This variant, currently classified as having no functional effect (Hofman classification; Table 2), was previously reported in association with hyperkalemic and normokalemic periodic paralysis. ${ }^{33,34}$ However, a functional expression study supported the hypothesis that this variant should be classified as a rare benign polymorphism rather than a causative mutation. ${ }^{35}$ No other family members were included in that study to evaluate segregation. The CLCN1 gene analysis was not performed because no patient consent was obtained. Therefore, we cannot confirm the involvement of SCN4A p.(Val781Ile) in the muscular phenotype of this patient.

Two of the three family members (I1 and I3) of the second SCN5Anegative family (I) had myotonic discharges on EMG and a novel c.3901_3903delCAG (p. (Gln1301del)) variant in the intracellular domain 3-4 of SCN4A. The localization of this amino-acid deletion as well as the phenotype/genotype concordance in the family members advocates the probable function affecting the role of this genetic change.

In the third Brugada family (J), positive for the known SCN5A variant c.2632C > T p.(Arg878Cys), ${ }^{32}$ no SCN4A mutation was found in the index patient (J1) who presented myotonic features on EMG. Consequently, the CLCN1 gene was analyzed. All the family members with myotonic features on EMG (J1, J2, J7, J8 and J12) are heterozygous carriers of a c.774+1G $>$ A variant in intron 6 , which has not been described before. In this large family, there was a $100 \%$ genotype-phenotype concordance for myotonia as none of the myotonia-negative members who were tested (J3, J4 and J9-J11) carried the CLCN1 variant, therefore confirming the causative role for this variant. Based on these findings, we can conclude that some family members, besides having a BS, suffer from
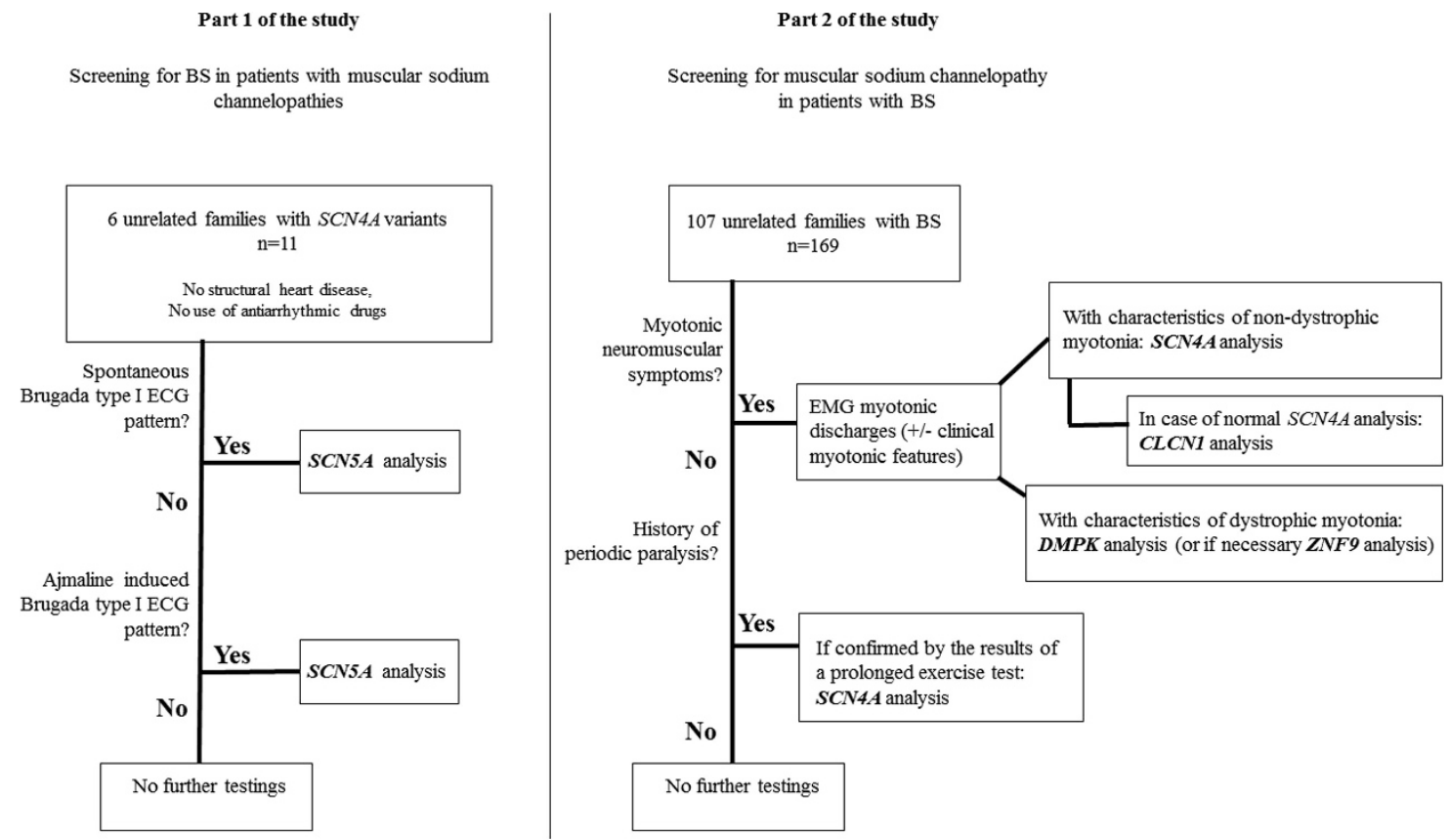

Figure 2 Study design. 


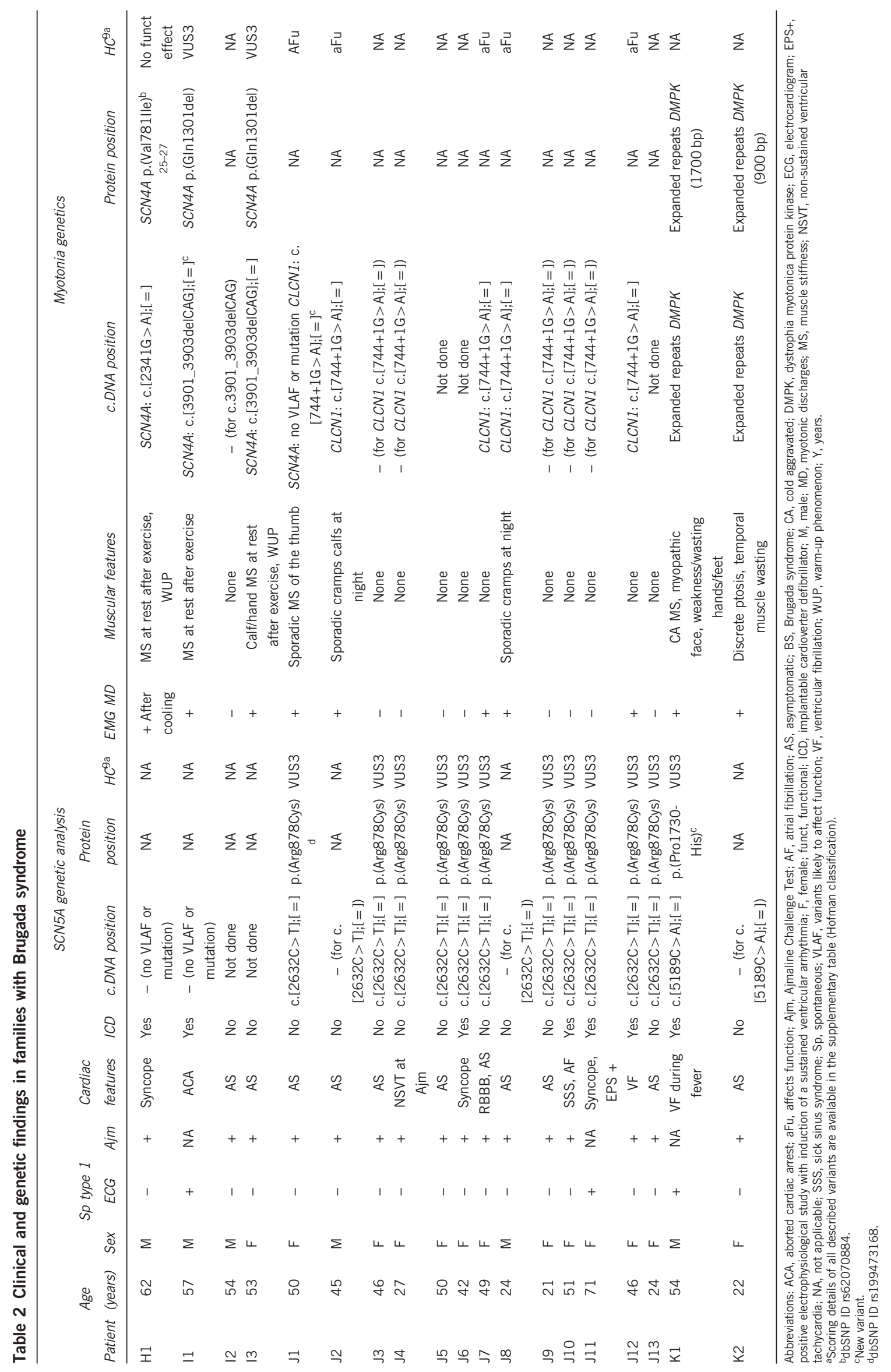




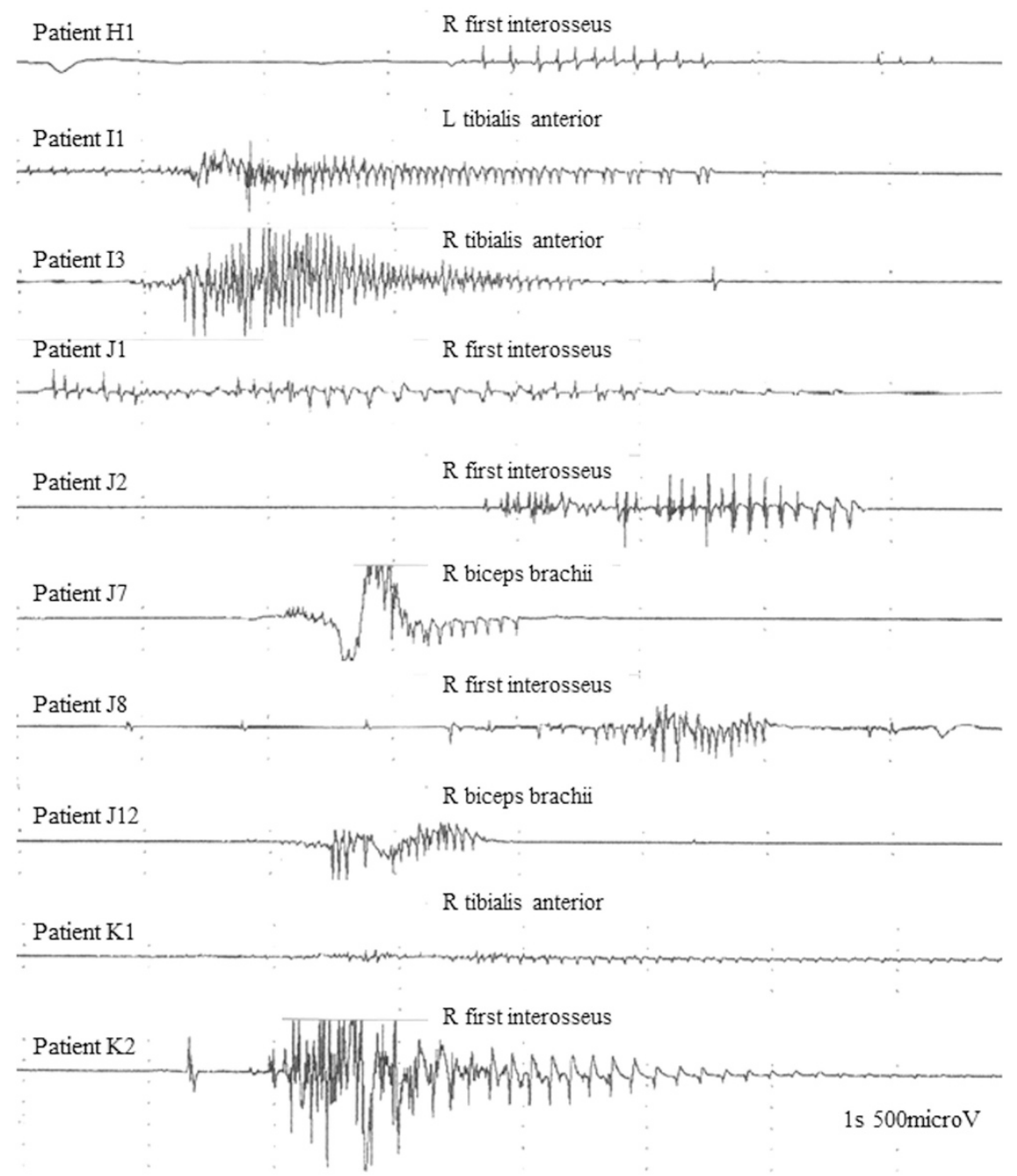

Figure 3 Myotonic discharges in electromyography of Brugada patients.

autosomal-dominant myotonia congenita (Thomsen disease). However, most of them were clinically asymptomatic or described minimal nonspecific muscular symptoms. Interestingly, not all the Brugada patients in this family have the known c.2632C $>\mathrm{T}$ p.(Arg878Cys) SCN5A variant. Some of them only have the CLCN1 variant (Table 2), do not display malignant cardiac events (J2 and J8), but have a positive Ajmaline Challenge Test in addition to their myotonic features.

The fourth family (K1 and K2) had typical clinical features of a myotonic dystrophy (DM1), including ptosis and atrophy of the temporal and sternocleidomastoid muscle. Discrete distal muscular weakness and percussion myotonia were only observed in the oldest patient (K1). His major complaints were cramps in both hands and grip myotonia, exacerbated by cold. The analysis of the DMPK gene (dystrophia myotonica protein kinase) showed an expansion of the CTG trinucleotide repeat in the $3^{\prime}$ end of the gene in both father (1700 bp) and daughter (900 bp), the presence of which confirmed the diagnosis of DM1 in both patients. In the cardiac symptomatic proband (K1), additional $S C N 5 A$ gene analysis revealed a novel variant c.5189C $>$ A (p.(Pro1730His)), which probably affects the function. His cardiac asymptomatic daughter (K2) did not carry this variant despite a positive Ajmaline Challenge Test. This finding demonstrates that in this family this SCN5A variant is not disease causing, as it not segregating correctly.

\section{DISCUSSION}

We report on three families (families A, G and I) with an SCN4Arelated NDM and one family $(\mathrm{H})$ with a possible $S C N 4 A$-related NDM in association with BS. Two out of five patients with predicted gain-offunction SCN4A variants, and BS, had cardiac malignant events (A1 and I1). Furthermore, some family members of two other SCN4A-positive families (B and D) displayed cardiac arrhythmia symptoms. One had palpitations and a short documented episode of atrial fibrillation (B3). The second had syncopes and a documented non-sustained ventricular tachycardia (D1). No function affecting SCN5A variant was found in their families. Although two case studies $^{5,6}$ suggested a possible role of $S C N 4 A$ in cardiac arrhythmogenesis, this association has not yet been documented.

Because SCN4A and SCN5A are not genetically linked to each other (chromosome $17 \mathrm{q} 23$ and $3 \mathrm{q} 12$, respectively), ${ }^{36}$ we need to take a closer look at the role and the localization of Nav1.4 channels in the human cardiac muscle to find a possible explanation for these findings. Distribution of voltage-gated sodium channels differs between different mammalian species. Although ischemic rat hearts 
do not express functional NaV1.4 channels, ${ }^{37}$ they are present in mouse, pig and human cardiac tissue. ${ }^{7}$ By staining the $\alpha$-subunit in human atrial tissue, Kaufmann and et al. ${ }^{8}$ demonstrated that the tetrodotoxin (TTX)-sensitive Nav1.4 and the non-TTX-sensitive Nav1.5 channels accounted for $2.6 \%$ and $87.7 \%$ of total sodium channel staining, respectively. The human ventricle also expresses the SCN4A $\alpha$-subunit gene, ${ }^{5}$ and in whole human heart samples, the transcript level of Nav1.4 was $1.1 \%{ }^{7}$ Unlike other $\alpha$ channels, both Nav1.4 and Nav1.5 are present in a striated pattern on the cell myocyte surface, in register with the z-lines. ${ }^{8}$ How Nav1.5 and Nav1.4 interact with each other is not clear, but their colocalization, which is also similar to the localization of Cav1.2 calcium channels in atrial tissue, could ensure rapid activation of the calcium channels and thus contraction. Whether SCN $4 A$ mutations, resulting in dysfunctional Nav1.4 channels, could influence this interchannel interaction and result in a reduced net depolarization current, as seen in $\mathrm{BS}^{38,39}$ and/or aberrant calcium channel activation, needs further investigation including functional expression studies and wedge preparation models.

However, we should also emphasize that in contrast to the lossof-function of NaV1.5 sodium channels in the SCN5A-associated BS, muscular sodium channelopathies with myotonic features typically result from a gain-of-function of Nav1.4 channels. Therefore, gain-offunction SCN4A mutations in cardiac tissue are less likely to result in a reduced net depolarization current, as seen in BS. In patients with PMC, prolonged QTc intervals were observed in association with a gain-of-function SCN4A mutation (p. $(\operatorname{Arg} 1448 C y s))^{5}$. Such repolarization abnormalities have also been seen in long QT syndrome type 3 (LQTS3) as a result of gain-of-function SCN5A mutations. ${ }^{40} \mathrm{We}$ were, however, unable to demonstrate prolonged QTc intervals in association with p.(Val781Ile), p.(Gln1301del), p.(Leu1436Pro), p.(Arg1448Cys), p.(Val1458Asp) and p.(Arg1460Gln). In LQTS3, prolongation of the QT interval is expected to result from decreased $\mathrm{K}^{+}$repolarization currents, increased $\mathrm{Ca}^{2+}$ entry or a sustained entry of $\mathrm{Na}^{+}$(late $\mathrm{I}_{\mathrm{Na}}$ ) into the cardiomyocyte. ${ }^{40}$ Whether a parallelism can be drawn for SCN4A and if SCN4A mutations might result in overlapping clinical properties of different syndromes such as BS and LQTS3 ${ }^{41}$ requires further investigation.

Our data support the utility of a cardiac work-up in patients with SCN4A mutation or variants. Class Ic antiarrhythmic drugs such as flecainide and propafenone, which are used for the symptomatic treatment of myotonia, are contraindicated in Brugada syndrome. ${ }^{42,43}$ Caution during a challenge test is therefore required. In contrast, Mexiletine, a class Ib antiarrhythmic, is considered to be safe in reducing myotonia ${ }^{44}$ and is known to restore trafficking defects in BS. ${ }^{45,46}$

More intriguing is the presence of a rare pathology such as Thomsen disease $(<1 / 100000)$ in one BS family $(\mathrm{J})$. It is caused by an $C L C N 1$ variant encoding for the $\mathrm{ClC}-1$ voltage-gated chloride channel. ${ }^{4}$ Several chloride channels in the heart, including ClC-2 and ClC-3 voltage-gated chloride channels, have been previously reported as contributors to arrhythmogenesis. ${ }^{47}$ Only recently, the expression of CLCN1 mRNA transcripts has been reported in the human brain and heart. ${ }^{48}$ Therefore, no clear explanation can be found for the concomitant occurrence of both diseases.

DM1 was diagnosed in one family (K). In recent publications, ${ }^{49,50}$ a significantly high frequency of spontaneous and ajmaline-induced type 1 Brugada ECG pattern was detected in DM1 patients (prevalence: $0.5-3 \%$ ), suggesting that our findings are not a coincidence. Patient K1 presented with a VF episode during a febrile episode, a typical phenomenon seen in BS. A possible explanation underlying the link between DM1 and BS could be that the CTG repeats in DMPK result in the accumulation of nuclear mRNA sequences, facilitating abnormal splicing of several genes such as SCN5A. ${ }^{49}$ Others suggest that because both DM1 and BS are associated with focal fibrosis and fatty infiltration, ${ }^{51,52}$ their coexistence could amplify the subsequent arising conduction delay in the right ventricle (RV). Such RV delay has been thought responsible for the arrhythmogenesis in BS. ${ }^{39}$

The 'pure' self-sufficient causative role of loss-of-function mutations of the SCN5A gene in the Brugada ECG pattern or syndrome has been challenged and BS is no longer thought to be a pure monogenic disorder. Phenotype-positive genotype-negative family members (genotype-phenotype discordance), SCN5A mutation carriers without a spontaneous or induced type 1 BS ECG pattern (incomplete penetrance) and family members who carry the same SCN5A mutation and express wide-ranging clinical manifestations (variable expression) support this statement. ${ }^{53}$

Only about $35 \%$ of BS patients have been determined to have a genetic cause and nearly $30 \%$ carry a mutation in SCN5A. Besides the known mutations in several genes (SCN5A, GPD1L, SCN1B, SCN2B, SCN3B, MOG1, RANGRF, SLMAP, KCNE3, KCNJ8, HCN4, KCNE5, KCND3, CACNA1C, CACNB2, CACNA2D1, SCN1OA and TRPM4), ${ }^{2,3}$ other factors also have an important role in the resulting phenotype such as additional variants $^{54}$ (compound heterozygous disease-associated polymorphisms in family A), ${ }^{15,16,55}$ epigenetic mechanisms (DNA methylation, posttranslational modifications and RNA mechanisms) ${ }^{56}$ and phenotype modulators (vagal tone, sex hormones and febrile status). ${ }^{57}$ It is likely that these additional factors influence the precise phenotypic expression and are therefore responsible for phenotypic overlap ${ }^{41}$ and variable expressivity or incomplete penetrance $^{58}$ as seen in these families. As described for SCN10A, which encodes the sodium channel isoform Nav1.8, ${ }^{54}$ the presence of an SCN $4 A$ variant or a CLCN1 variant or the expansion of a CTG trinucleotide repeat in $D M P K$, is probably not solely responsible for an arrhythmic event, but would rather act as an additional modifier. Further studies, including genome-wide association studies, are required to further assess the influence of these additional variants on conduction and BS.

In conclusion, we report a high number of patients with coexisting BS and SCM. Our findings suggest a possible impact of SCN4A variants on the pathophysiological mechanism underlying the development of a type 1 ECG pattern and of malignant arrhythmia symptoms in some patients with BS.

\section{CONFLICT OF INTEREST}

The authors declare no conflict of interest. Prof Dr Pedro Brugada received grants and speaker fees from Biotronik, Medtronic, St Jude Medical and Boston Scientific. These disclosures are not related to the study.

\section{ACKNOWLEDGEMENTS}

We thank Richard Alloway and Orville Small for their thorough revision of the manuscript as native English speakers. We also thank the BRIGHTcore, a Vrije Universiteit Brussel and Université Libre de Bruxelles (VUB-ULB) interuniversity genomicscore facility for the BS gene panel analysis.

\footnotetext{
1 George Jr AL: Inherited disorders of voltage-gated sodium channels. J Clin Invest 2005; 115: 1990-1999.

2 Kapplinger JD, Tester DJ, Alders $\mathrm{M}$ et al: An international compendium of mutations in the SCN5A-encoded cardiac sodium channel in patients referred for Brugada syndrome genetic testing. Heart Rhythm 2010; 7: 33-46.

3 Nielsen MW, Holst AG, Olesen S-P, Olesen MS: The genetic component of Brugada syndrome. Front Physiol 2013; 4: 179.

4 Horga A, Raja Rayan DL, Matthews E et al: Prevalence study of genetically defined skeletal muscle channelopathies in England. Neurology 2013; 80: 1472-1475.
} 
5 Péréon $\mathrm{Y}$, Lande G, Demolombe $\mathrm{S}$ et al: Paramyotonia congenita with an SCN4A mutation affecting cardiac repolarization. Neurology 2003; 60: 340-342.

6 Maffè S, Signorotti F, Perucca A et al: Atypical arrhythmic complications in familial hypokalemic periodic paralysis. J Cardiovasc Med (Hagerstown) 2009; 10: 68-71.

7 Blechschmidt S, Haufe V, Benndorf K, Zimmer T: Voltage-gated $\mathrm{Na}^{+}$channel transcript patterns in the mammalian heart are species-dependent. Prog Biophys Mol Biol 2008; 98: 309-318.

8 Kaufmann SG, Westenbroek RE, Maass AH et al: Distribution and function of sodium channel subtypes in human atrial myocardium. J Mol Cell Cardiol 2013; 61: 133-141.

9 Hofman N, Tan HL, Alders M et al: Yield of molecular and clinical testing for arrhythmia syndromes: report of 15 years' experience. Circulation 2013; 128: 1513-1521.

10 Wallis Y, Payne S, McAnulty C et al: Practice Guidelines for the Evaluation of Pathogenicity and the Reporting of Sequence Variants in Clinical Molecular Medicine. Guidelines updated by the Association for Clinical Genetic Science (ACGS) and the Dutch Society of Clinical Genetical Laboratory Specialists (VKGL) (approved September 2013). Universitair Medisch Centrum: St Radboud.

11 Fournier E, Arzel M, Sternberg D et al: Electromyography guides toward subgroups of mutations in muscle channelopathies. Ann Neurol 2004; 56: 650-661.

12 Fournier E, Viala K, Gervais $\mathrm{H}$ et al: Cold extends electromyography distinction between ion channel mutations causing myotonia. Ann Neurol 2006; 60: 356-365.

13 Matthews E, Tan SV, Fialho D et al: What causes paramyotonia in the United Kingdom? Common and new SCN4A mutations revealed. Neurology 2008; 70: 50-53.

14 Bissay V, Keymolen K, Lissens W, Laureys G, Schmedding E, De Keyser J: Late onset painful cold-aggravated myotonia: three families with SCN4A L1436P mutation. Neuromuscul Disord 2011; 21: 590-593.

15 Shinlapawittayatorn K, Du XX, Liu H, Ficker E, Kaufman ES, Deschênes I: A common $S C N 5 A$ polymorphism modulates the biophysical defects of SCN5A mutations. Heart Rhythm 2011; 8: 455-462.

16 Gouas L, Nicaud V, Berthet M et al: Association of KCNQ1, KCNE1, KCNH2 and SCN5A polymorphisms with QTC interval length in a healthy population. Eur J Hum Genet 2005; 13: 1213-1222.

17 Kim DS, Kim EJ, Jung DS et al: A Korean family with Arg1448Cys mutation of SCN4A channel causing paramyotonia congenita: electrophysiologic, histopathologic, and molecular genetic studies. J Korean Med Sci 2002; 17: 856-860.

18 Bissay V, Keymolen K, Lissens W, Schmedding E, De Keyser J: A novel mutation in the $S C N 4 A$ responsible for cold-induced myotonia with normal electromyography findings on room temperature. J Neurol Sci 2011; 308: 162-164.

19 Priori SG, Wilde AA, Horie $M$ et al: Executive summary: HRS/EHRA/APHRS expert consensus statement on the diagnosis and management of patients with inherited primary arrhythmia syndromes. Europace 2013; 15: 1389-1406.

20 Rolf S, Bruns HJ, Wichter T et al: The ajmaline challenge in Brugada syndrome: diagnostic impact, safety, and recommended protocol. Eur Heart J 2003; 24: 1104-1112.

21 Ackerman MJ, Marcou CA, Tester DJ: Personalized medicine: genetic diagnosis for inherited cardiomyopathies/channelopathies, Rev Esp Cardiol 2013; 66: 298-307.

$22 \mathrm{Li} \mathrm{H}$, Durbin R: Fast and accurate short read alignment with Burrows-Wheeler transform. Bioinformatics 2009; 25: 1754-1760.

23 Picard-tools - A set of tools for working with next generation data in the BAM format. Available at: http://broadinstitute.github.io/picard (version 1.97) (last accessed 26 August 2013).

$24 \mathrm{Li} \mathrm{H}$, Handsaker B, Wysoker A et al: The sequence alignment/map format and SAMtools. Bioinformatics 2009; 25: 2078-2079.

25 McKenna A, Hanna M, Banks E et al: The genome analysis toolkit: a mapreduce framework for analyzing next-generation DNA sequencing data. Genome Res 2010; 20: 1297-1303.

26 Antzelevitch C, Brugada P, Borggrefe $\mathrm{M}$ et al: Brugada syndrome: report of the second consensus conference. Heart Rhythm 2005; 2: 429-440.

27 Trip J, Drost G, Verbove DJ et al: In tandem analysis of CLCN1 and SCN4A greatly enhances mutation detection in families with non-dystrophic myotonia. Eur J Hum Genet 2008; 16: 921-929.

28 Brook JD, McCurrach ME, Harley HG et al: Molecular basis of myotonic dystrophy: expansion of a trinucleotide (CTG) repeat at the $3^{1}$ end of a transcript encoding a protein kinase family member. Cell 1992; 68: 799-808.

29 Warner JP, Barron LH, Goudie D et al: A general method for the detection of large CAG repeat expansions by fluorescent PCR. J Med Genet 1996; 33: 1022-1026.

30 Junttila MJ, Raatikainen MJ, Karjalainen J, Kauma H, Kesäniemi YA, Huikuri HV Prevalence and prognosis of subjects with Brugada-type ECG pattern in a young and middle-aged Finnish population. Eur Heart J 2004; 25: 874-878.

31 Gui J, Wang T, Jones RP, Trump D, Zimmer T, Lei M: Multiple loss-of-function mechanisms contribute to SCN5A-related familial sick sinus syndrome. PLOS One 2010; 5: e10985.

32 Zhang $\mathrm{Y}$, Wang T, Ma A et al: Correlations between clinical and physiological consequences of the novel mutation R878C in a highly conserved pore residue in the cardiac $\mathrm{Na}^{+}$channel. Acta Physiol (Oxf) 2008; 194: 311-323.
33 Baquero JL, Ayala RA, Wang J et al: Hyperkalemic periodic paralysis with cardiac dysrhythmia: a novel sodium channel mutation? Ann Neurol 1995; 37: 408-411.

34 Xiuhai G, Weiping W, Ke Z, Hongbin W, Yiling S, Yanling M: Mutations of sodium channel alpha-subunit genes in Chinese patients with normokalemic periodic paralysis. Cell Mol Neurobiol 2008; 28: 653-661.

35 Green DS, Hayward LJ, George Jr AL, Cannon SC: A proposed mutation, Val781lle, associated with hyperkalemic periodic paralysis and cardiac dysrhythmia is a benign polymorphism. Ann Neurol 1997; 42: 253-256.

$36 \mathrm{Yu} \mathrm{FH}$, Catterall WA: Overview of the voltage-gated sodium channel family. Genome Biol 2003; 4: 207

37 Anyukhovsky EP, Sosunov EA, Kryukova YN et al: Expression of skeletal muscle sodium channel (Nav1.4) or connexin32 prevents reperfusion arrhythmias in murine heart. Cardiovasc Res 2011; 89: 41-50.

38 Wilde AA, Postema PG, Di Diego JM et al: The pathophysiological mechanism underlying Brugada syndrome: depolarization versus repolarization. J Mol Cell Cardiol 2010; 49: 543-553.

39 Meregalli PG, Wilde AA, Tan HL: Pathophysiological mechanisms of Brugada syndrome: depolarization disorder, repolarization disorder, or more? Cardiovasc Res 2005; 67: 367-378.

40 Wilde AA, Brugada R: Phenotypical manifestations of mutations in the genes encoding subunits of the cardiac sodium channel. Circ Res 2011; 108: 884-897.

41 Remme CA, Wilde AA, Bezzina CR: Cardiac sodium channel overlap syndromes: different faces of SCN5A mutations. Trends Cardiovasc Med 2008; 18: 78-87.

42 Rosenfeld J, Sloan-Brown K, George Jr AL: A novel muscle sodium channel mutation causes painful congenital myotonia. Ann Neurol 1997; 42: 811-814.

43 Alfonsi E, Merlo IM, Tonini M et al: Efficacy of propafenone in paramyotonia congenita. Neurology 2007; 68: 1080-1081.

44 Statland JM, Bundy BN, Wang Y et al: Mexiletine for symptoms and signs of myotonia in nondystrophic myotonia: a randomized controlled trial. JAMA 2012; 308: 1357-1365.

45 Moreau A, Keller DI, Huang $\mathrm{H}$ et al: Mexiletine differentially restores the trafficking defects caused by two Brugada syndrome mutations. Front Pharmacol 2012; 3: 62.

46 Márquez MF, Salica G, Hermosillo AG et al: Ionic basis of pharmacological therapy in Brugada syndrome. J Cardiovasc Electrophysiol 2007; 18: 234-240.

47 Duan D: Phenomics of cardiac chloride channels: the systematic study of chloride channel function in the heart. J Physiol 2009; 587: 2163-2177.

48 Chen TT, Klassen TL, Goldman AM, Marini C, Guerrini R, Noebels JL: Novel brain expression of CIC-1 chloride channels and enrichment of $C L C N 1$ variants in epilepsy. Neurology 2013; 80: 1078-1085.

49 Wahbi K, Algalarrondo V, Bécane $\mathrm{HM}$ et al: Brugada syndrome and abnormal splicing of SCN5A in myotonic dystrophy type 1. Arch Cardiovasc Dis 2013; 106: 635-643.

50 Pambrun T, Bortone A, Bois $\mathrm{P}$ et al: Unmasked Brugada pattern by ajmaline challenge in patients with myotonic dystrophy type 1. Ann Noninvasive Electrocardiol 2015; 20 : 28-36.

51 Coronel R, Casini S, Koopmann TT et al: Right ventricular fibrosis and conduction delay in a patient with clinical signs of Brugada syndrome: a combined electrophysiological, genetic, histopathologic, and computational study. Circulation 2005; 112: 2769-2777.

52 Vignaux 0, Lazarus A, Varin J et al: Right ventricular MR abnormalities in myotonic dystrophy and relationship with intracardiac electrophysiologic test findings: initial results. Radiology 2002; 224: 231-235.

53 Probst V, Wilde AA, Barc J et al: SCN5A mutations and the role of genetic background in the pathophysiology of Brugada syndrome. Circ Cardiovasc Genet 2009; 2: 552-557.

54 Bezzina CR, Barc J, Mizusawa Y et al: Common variants at SCN5A-SCN1OA and HEY2 are associated with Brugada syndrome, a rare disease with high risk of sudden cardiac death. Nat Genet 2013; 45: 1044-1049.

55 Bezzina CR, Rook MB, Groenewegen WA et al: Compound heterozygosity for mutations (W156X and R225W) in SCN5A associated with severe cardiac conduction disturbances and degenerative changes in the conduction system. Circ Res 2003; 92: 159-168.

56 Webster AL, Yan MS, Marsden PA: Epigenetics and cardiovascular disease. Can J Cardiol 2013; 29: 46-57.

57 Liu M, Yang KC, Dudley Jr SC: Cardiac sodium channel mutations: why so many phenotypes? Nat Rev Cardiol 2014; 11: 607-615.

58 Robyns T, Nuyens D, Van Casteren L et al: Reduced penetrance and variable expression of SCN5A mutations and the importance of co-inherited genetic variants: case report and review of the literature. Indian Pacing Electrophysiol J 2014; 14: 133-149.

Supplementary Information accompanies this paper on European Journal of Human Genetics website (http://www.nature.com/ejhg) 